\title{
Modeling of Alcator C-Mod Divertor Baffling Experiments
}

D. P. Stotler, C. S. Pitcher ${ }^{1}$, C. J. Boswell ${ }^{1}$, T. K. Chung ${ }^{1}$, B. LaBombard ${ }^{1}$, B. Lipschultz ${ }^{1}$, J. L. Terry ${ }^{1}$, and R. J. Kanzleiter ${ }^{2}$

Princeton Plasma Physics Laboratory Princeton University

${ }^{1}$ MIT Plasma Science and Fusion Center, NW17, Cambridge, MA 02139

${ }^{2}$ Rennselaer Polytechnic Institute, Troy, NY 12181 (Presently at Los Alamos National Laboratory, Los Alamos, NM, USA)

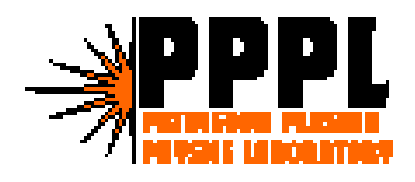

Note: This poster will be made available on the Web at:

http: / /w3 - pppl -gov/degas 2 /

within two weeks of the end of the conference. 


\section{INTRODUCTION}

- C-Mod divertor baffling experiments:

- Change divertor - main chamber conductance by 2 ,

- Divertor neutral pressure also changes by 2 .

- Infer that bypass current is constant!

- Divertor plasma conditions and $\mathrm{D}_{\alpha}$ do not change!

- Implies:

- Atomic processes limit flow through bypass,

- Divertor behaves as if it were completely open.

- Modeling requires:

- Detailed treatment of geometry,

- Kinetic treatment of neutrals.

- $\Rightarrow$ use DEGAS 2 (Stotler 1992).

- Absence of effect on divertor plasma allows fixed plasma to be used,

- Use Langmuir probe data,

- And Two-Point model (Pitcher 1997).

- Find:

- Insenstivity of bypass current for large conductances,

- Dependence of pressure on conductance,

- No change in $\mathrm{D}_{\alpha}$.

- However, quantitative differences arise.

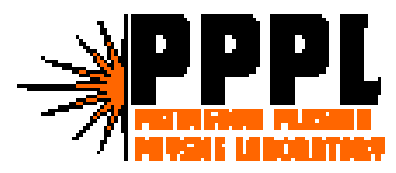




\section{Alcator C-Mod Divertor Bypass}

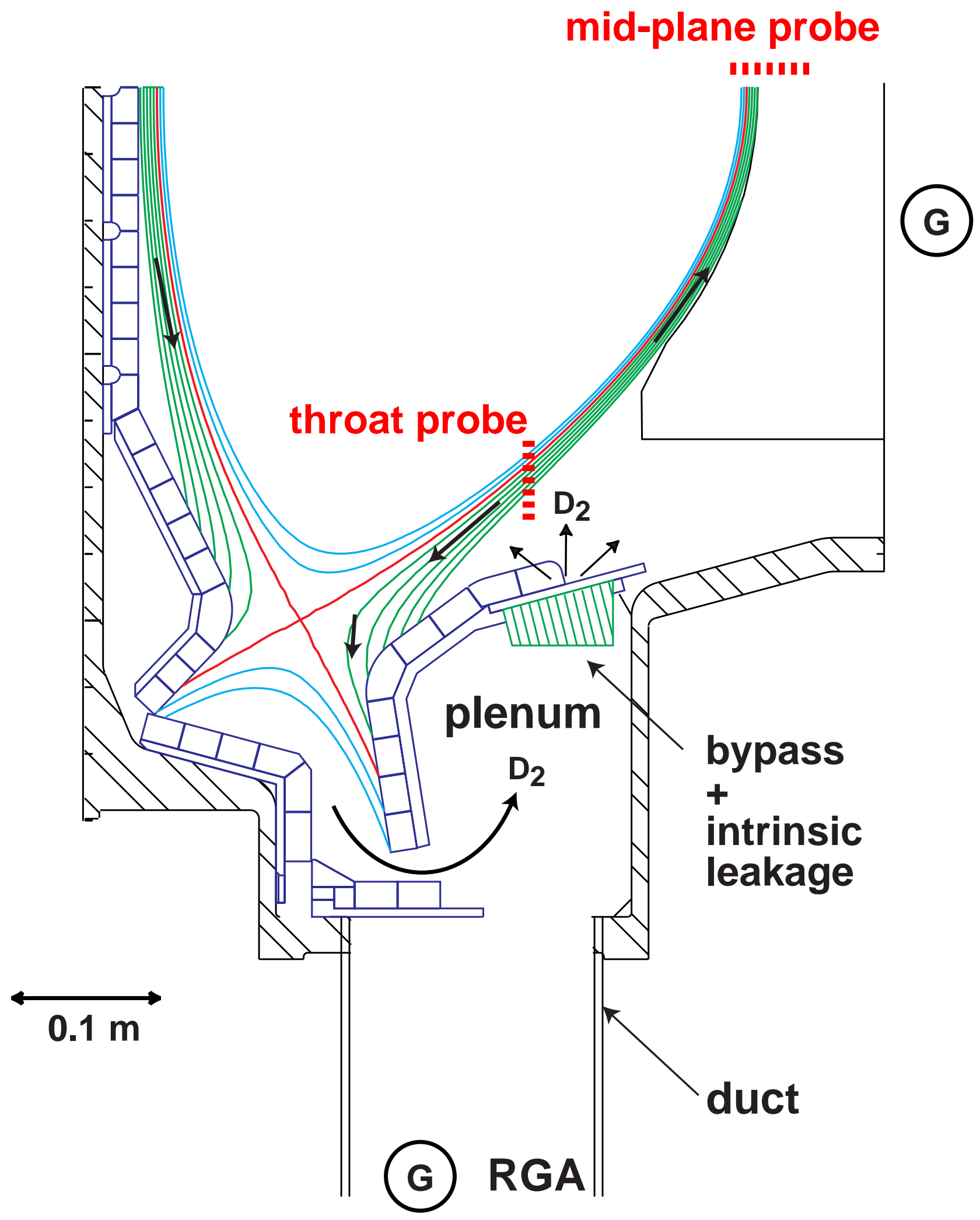


Effect of Bypass on $D_{2}$ Pressures

$1 \mathrm{mTorr} \sim 0.1 \mathrm{~Pa}=10^{-3} \mathrm{mbar}$

(a) D2 mid-plane pressure (mTorr)

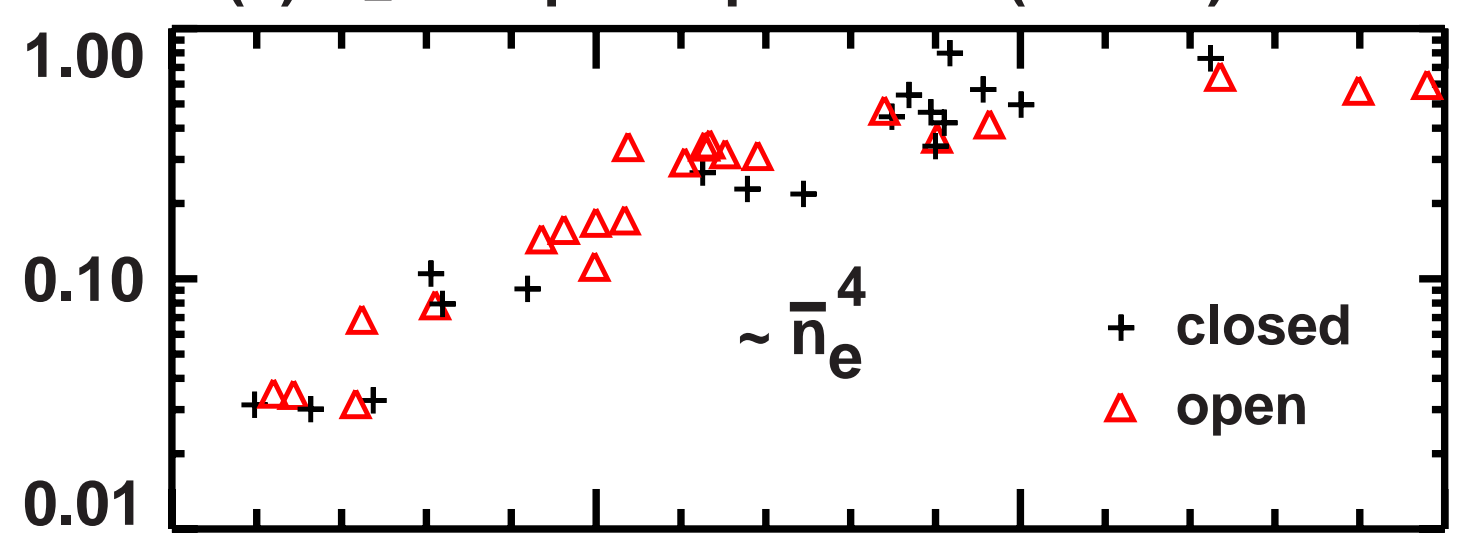

(b) $\mathrm{D}_{2}$ divertor pressure (mTorr)
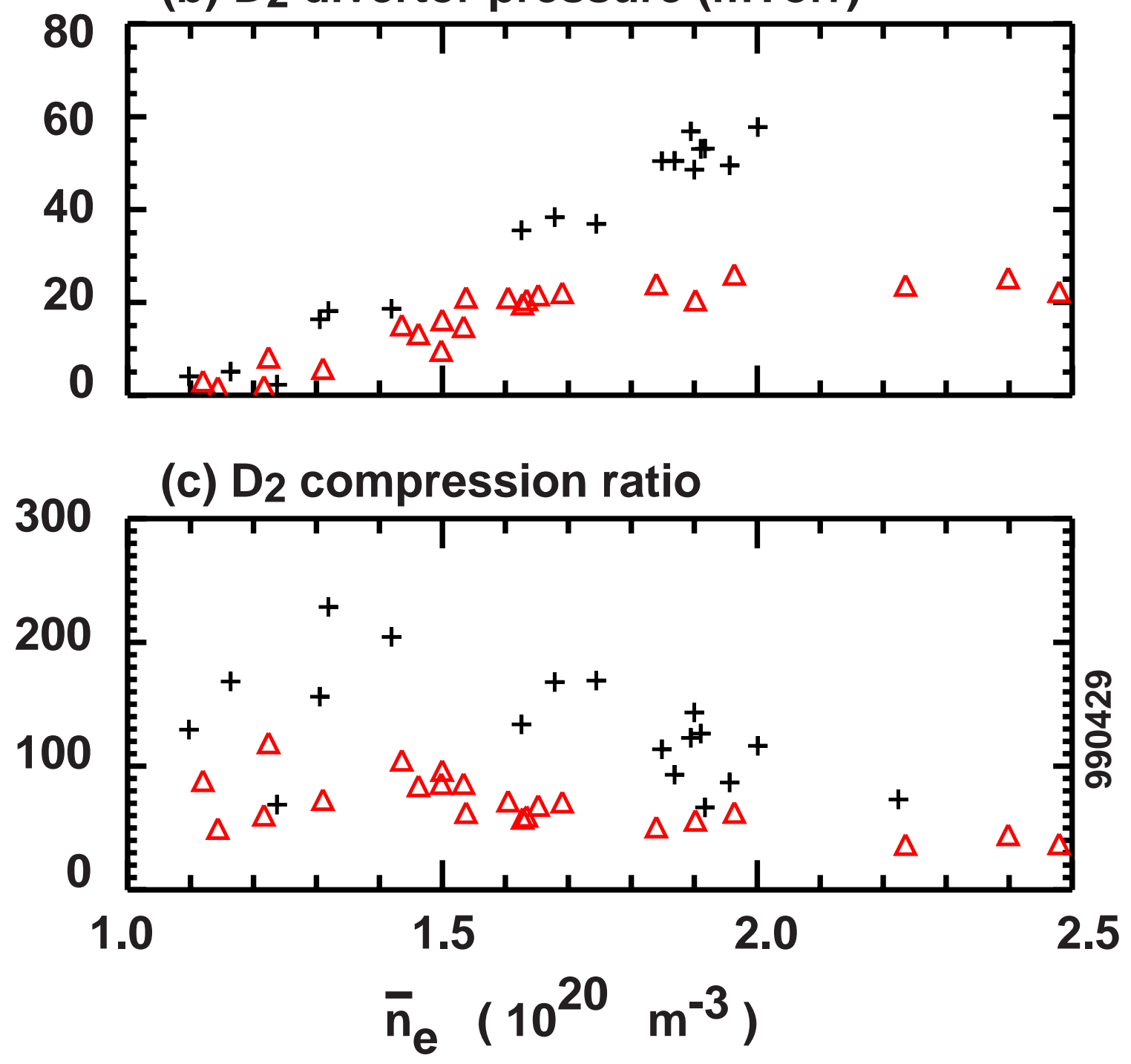


\section{Leakage Flux is comparable to ion flux to outer plate}

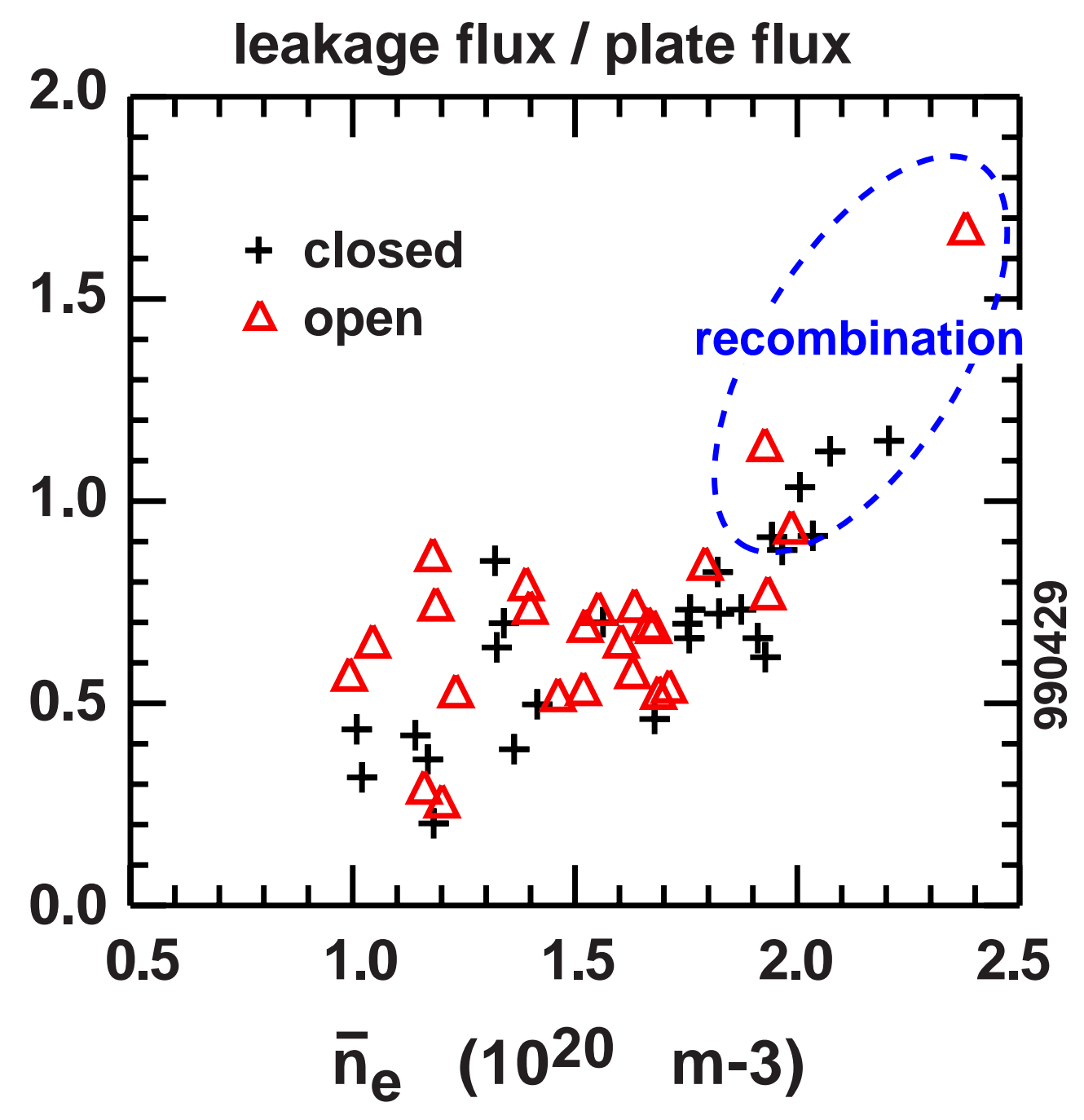

this is surprising given that the C-Mod baffling is relatively closed, $f \sim 0.15$ 


\section{EXPERIMENTAL DATA}

- Divertor bypass experiments described in (Pitcher 2000).

- Focus on shot 990429019, $t=0.95 \mathrm{~s}$,

$-\bar{n}_{e}=1.46 \times 10^{20} \mathrm{~m}^{-3}$,

- Both targets in high recycling regime,

$-P_{\text {div }}=15$ mTorr with bypass open,

$-P_{\text {div }}=30$ mTorr with bypass closed.

- Plasma data from scanning probes at midplane \& throat,

- Target data and fluxes from fixed Langmuir probes.

- Compare with divertor viewing $\mathrm{D}_{\alpha}$ array

- B-top, 63 detectors.

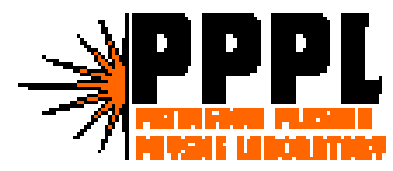


- Plasma for DEGAS 2:

- 1-D "Two Point" model for variation between probes,

- Plasma pressure constant along flux surfaces,

- Except in recycling region near target,

- Pressure drops to target value,

- Size estimated for these simulations.

- Parameters in PFR interpolated between inner \& outer values.

- $n_{e}=n_{i}, T_{e}=T_{i}$, no impurities.

- Plasma outside computational mesh:

$* 4 \mathrm{~cm}$ radial density decay,

* constant $T$.

- Main chamber source:

- Simulate recycling on limiters in main chamber (Umansky 1998),

- Calculate using

$$
\Gamma=\frac{1}{4} n_{D_{2}} \sqrt{\frac{8 T_{\text {wall }}}{\pi m}},
$$

- With $n_{D_{2}}=P_{\text {main }} / T_{\text {wall }}$,

- Use measured value $P_{\text {main }}=0.15$ mTorr. 


\section{DESCRIPTION OF SIMULATION}

- Geometry

- Outline of vacuum vessel, including

* Divertor plenum,

* Lower port,

$*$ RF limiter.

- EFIT equilibrium,

- Loaded into DG,

- Generate plasma mesh with CARRE,

- Transfer "elements" and plasma mesh to definegeometry2d,

- Polygons broken up into triangles with Triangle (Shewchuk 1996),

- Polygons labeled with zone number,

- Converted to DEĢAS 2's internal "surfaces" and "cells".

- Conductance between divertor, duct, and plenum approximate.

- Bypass width $w$,

$-w=16 \mathrm{~mm} \rightarrow$ integrated area of $0.075 \mathrm{~m}^{2}$,

- Corresponds to bypass closed.

- With bypass open, total area estimated $0.150 \mathrm{~m}^{2}$, $\Rightarrow w=32 \mathrm{~mm}$,

- Also consider $w=0,8$, and $64 \mathrm{~mm}$.

$-w=0 \leftrightarrow$ ideal of closed divertor. 


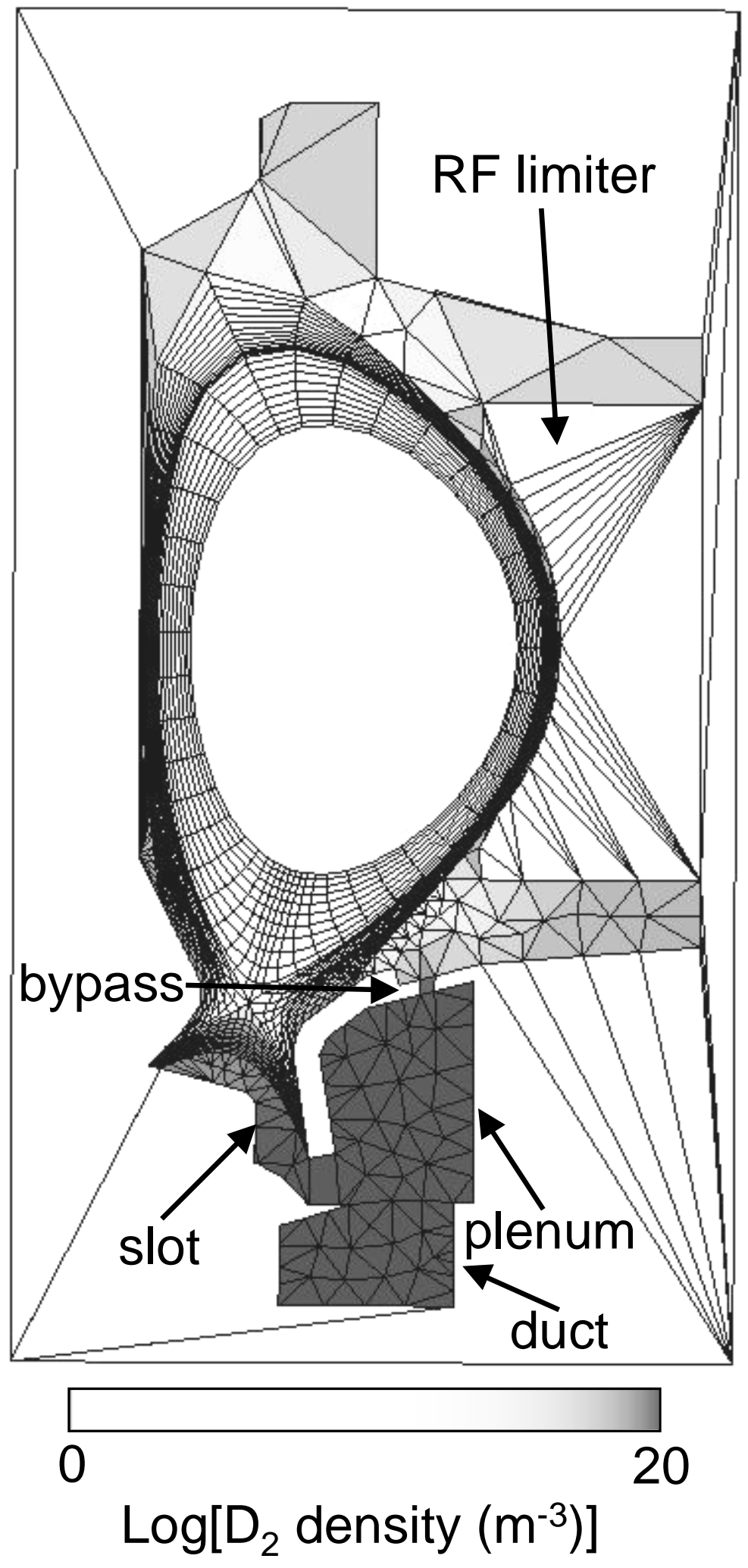

Fig. 1 

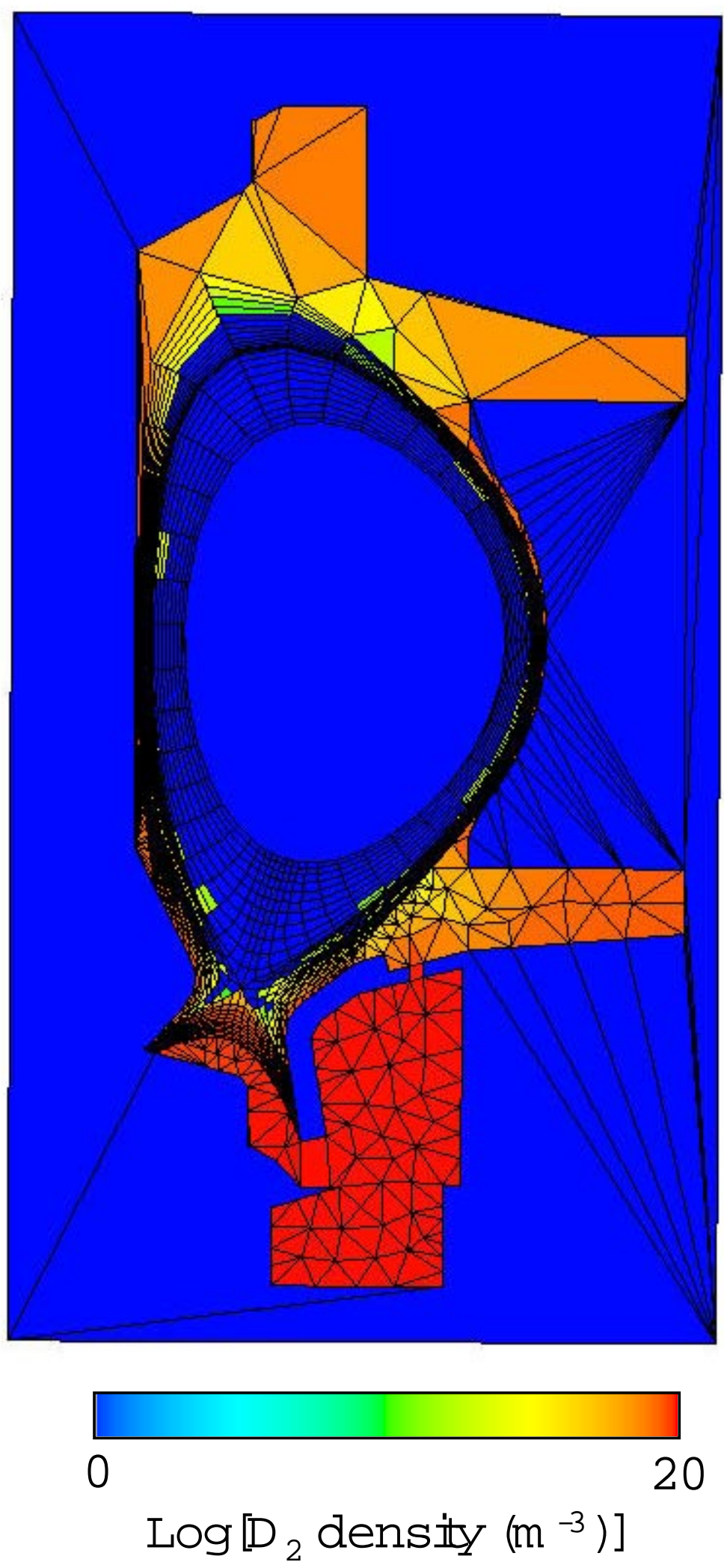
- All surfaces assumed to be molybdenum,

- Reflection coefficients from TRIM, 0.5-0.6.

- Non-reflected atoms desorb as thermal molecules.

- Atomic Physics

- Collisional-radiative model for

D ionization \& recombination,

* Based on (Weisheit 1975),

* Cross sections taken from (Janev 1993),

* Optically thin,

* Assess opacity effects later

using escape factor (e.g., Terry 1998).

- Molecular rates and kinetics as in (Stotler 1996), $*$ No $\mathrm{D}_{\alpha}$ from molecules ( $10 \%$ effect).

- Ion-neutral scattering,

* Differential cross sections computed using quantum mechanical techniques (Krstic 1998),

* $\mathrm{D}+\mathrm{D}^{+}$incorporates $\mathrm{CX} \&$ elastic scattering, $*$ Include $\mathrm{D}_{2}+\mathrm{D}^{+}$,

* Enforce minimum scattering angle,

- But constrain momentum transport to not change (Kanzleiter 1999),

* Use cumulative probability tables for cosine of scattering angle (Kanzleiter 1999).

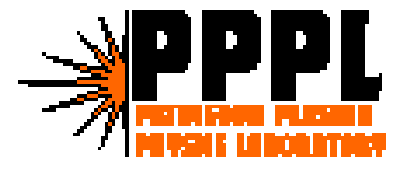


- Neutral-neutral elastic scattering, * BGK treatment just as in (Reiter 1997), * Knudsen numbers,

- 0.01 for molecules in plenum,

$\cdot>1$ for atoms in slot,

. $\Rightarrow$ Need nonlinear kinetic treatment.

- Running on 18 processor PC cluster (Stotler 2000), * Single iterations $\sim$ few minutes, * Few to several iterations required.

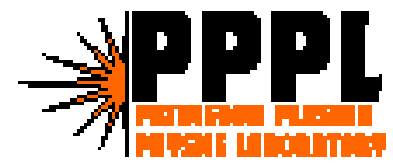




\section{RESULTS}

- Plot plenum pressure $P$ and bypass current $\phi$ vs. $w$,

- $\phi \ll$ ion current to target $=1.60 \times 10^{22} \mathrm{~s}^{-1}$

- Compare $\mathrm{D}_{\alpha}$ with measurements,

- Simulation results show no dependence on $w$,

- Emissions dominated by regions far from slot,

- And because $\phi$ small.

- Plenum pressures $\sim$ order of magnitude too small,

- $\mathrm{D}_{\alpha}$ is a factor of $3-10$ too small,

- Possible explanations:

- No reason to suspect Langmuir probes off by $>2$, $*$ But, earlier neutral particle balance encountered similar difficulties (Niemczewski 1995).

- Recombination in private flux region * $\mathrm{D}_{\gamma}$ tomography indicates more than obtained with simple plasma model for PFR, * Assess effect by adjusting plasma to match peak,

- Recombination source $\sim$ outer target current,

- $\mathrm{D}_{\alpha}$ plotted,

- $P \uparrow$ from 0.97 mTorr ("closed" bypass) to 1.88 .

- Recombination nearer outer target may have larger effect.

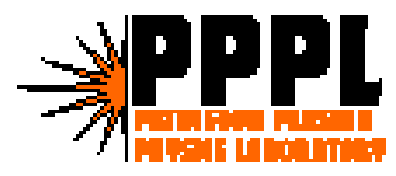



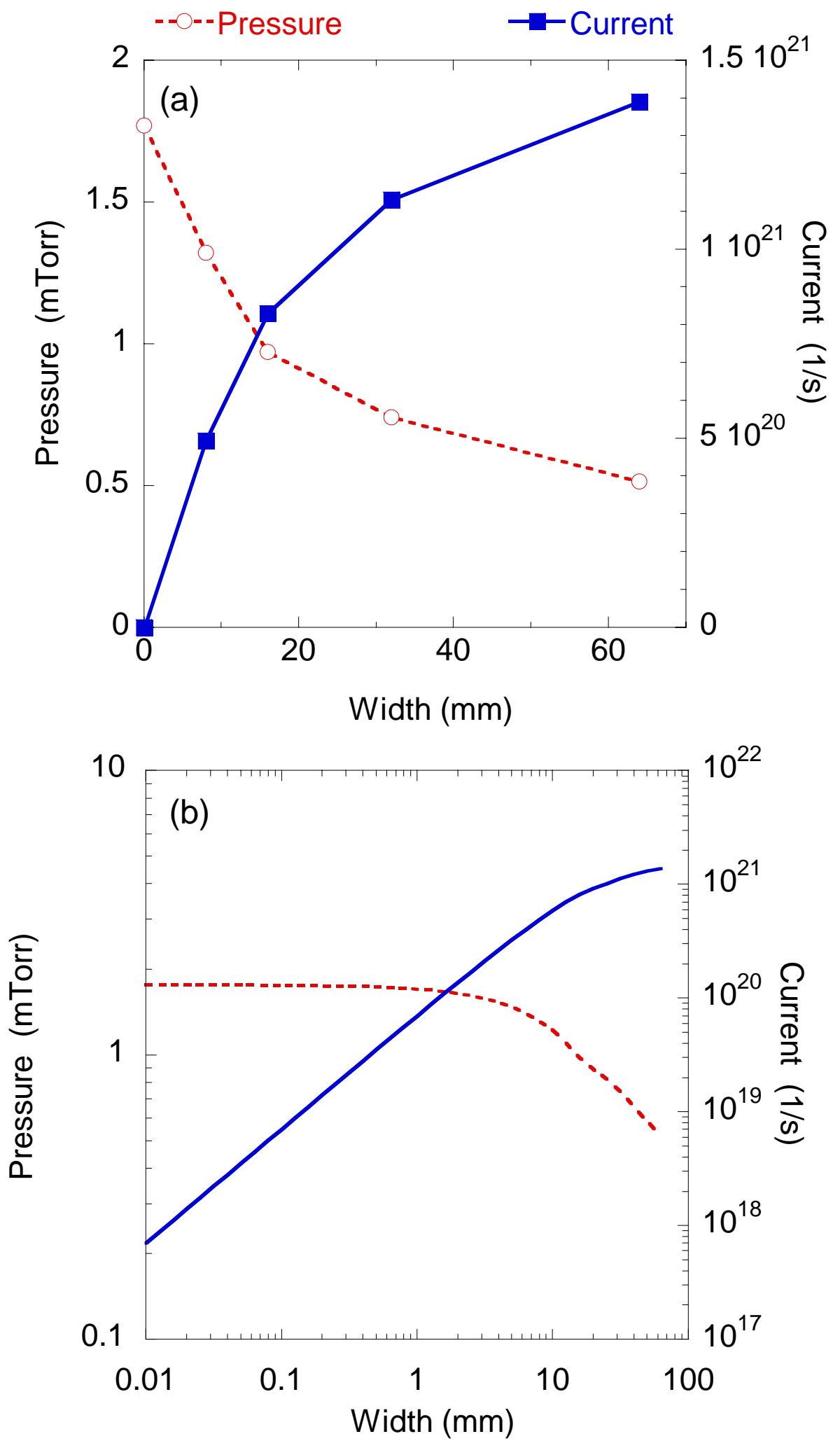

Fig. 2 


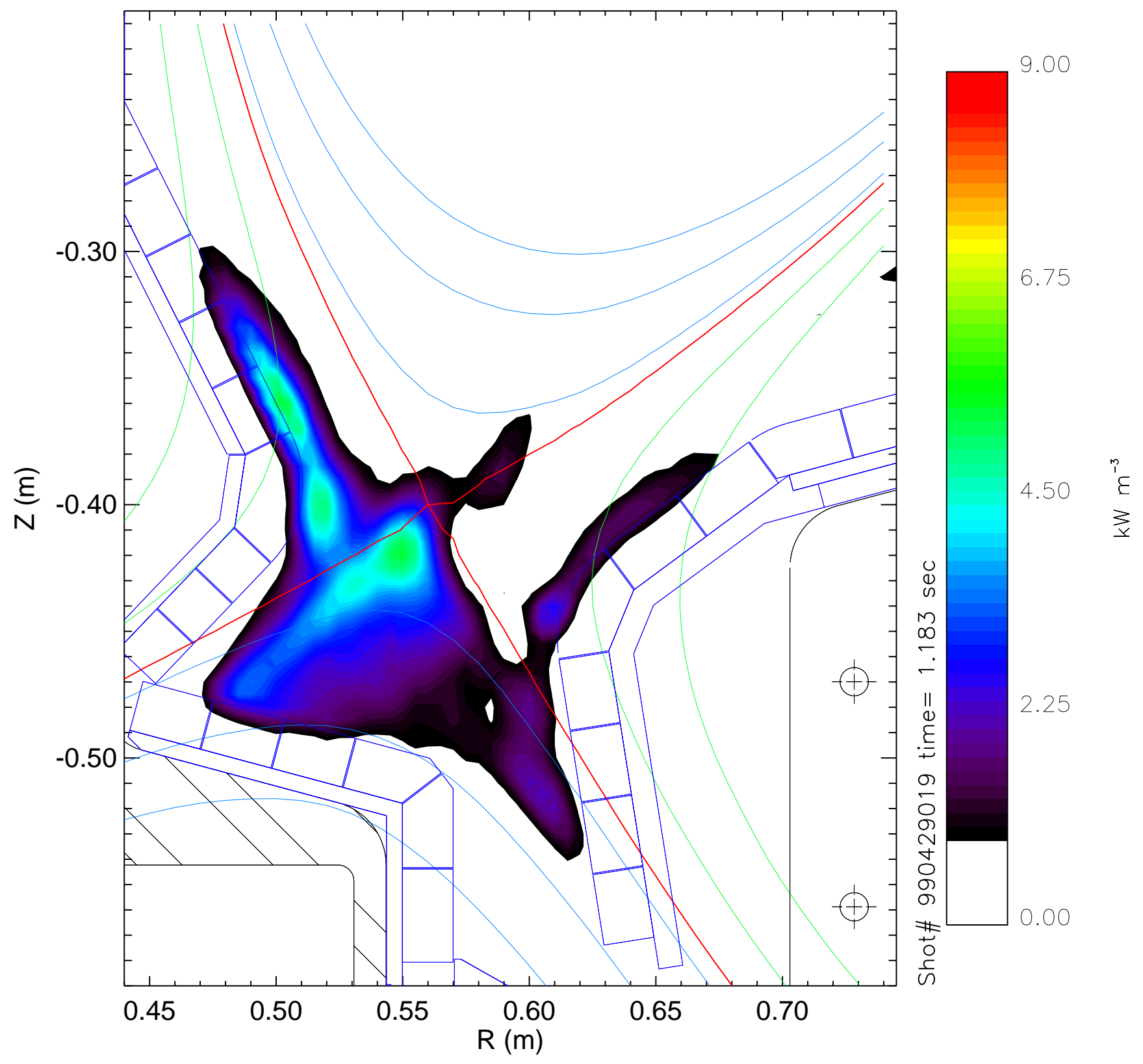




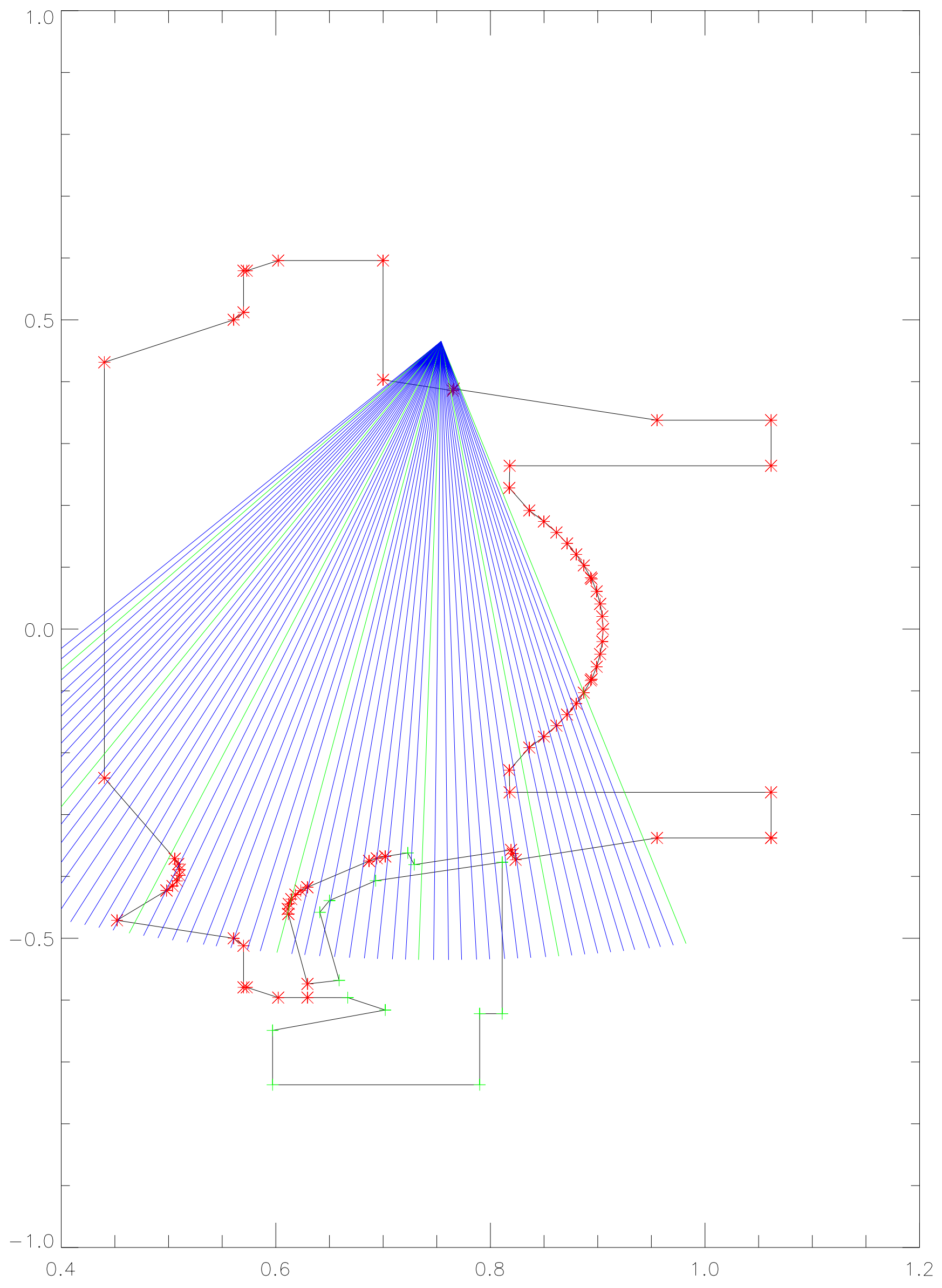




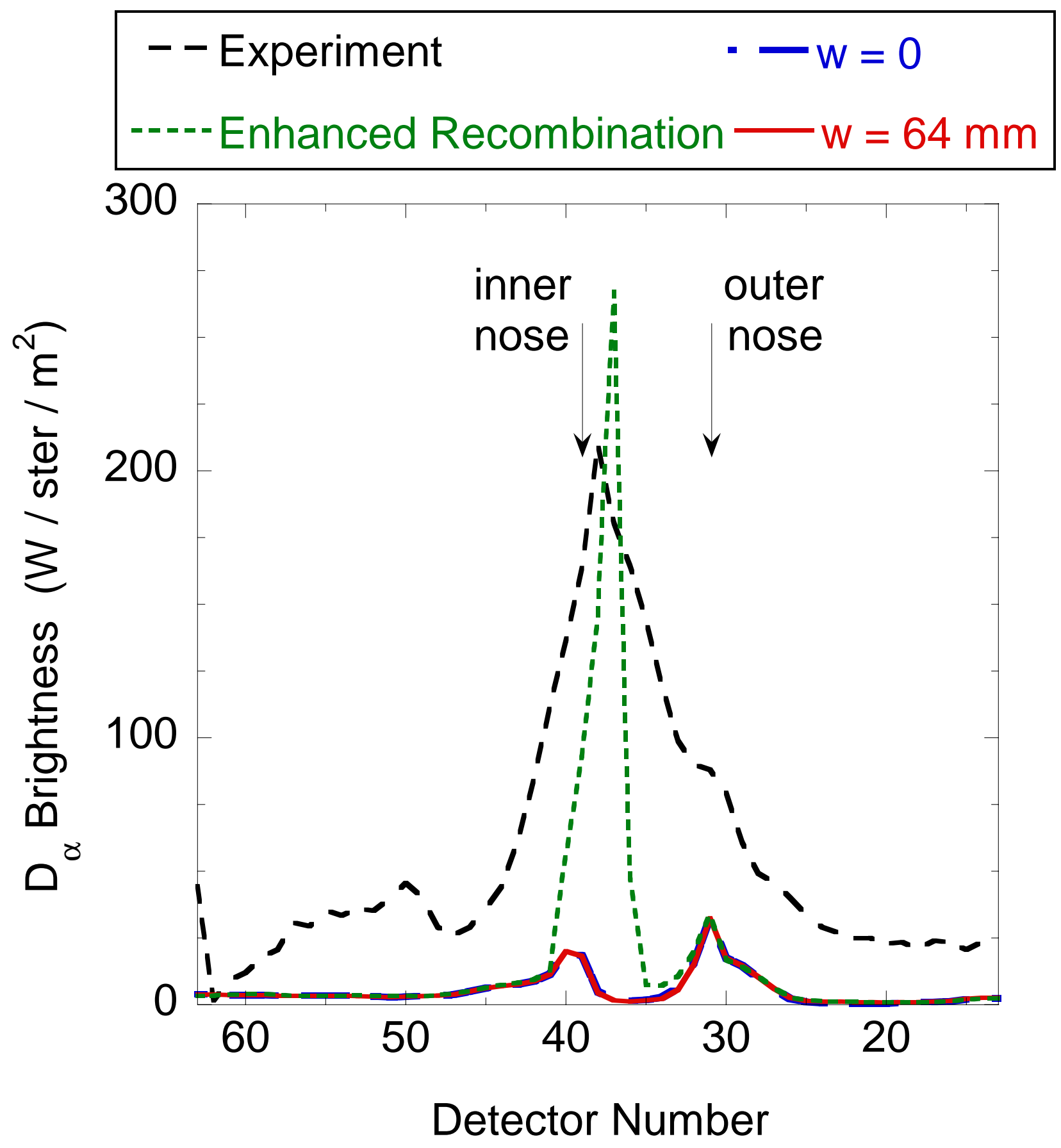


- Treatment of recycling region, * Size of region and density peak only estimated, * Errors could overemphasize ionization, * Should iterate DEGAS 2 \& plasma model, * Bound magnitude of effect by capping $n$ and $T$ at $1 \times 10^{20} \mathrm{~m}^{-3}$ and $4 \mathrm{eV}$,

- $P$ and $\phi \uparrow$ by 2 ,

- $\mathrm{D}_{\alpha}$ less affected.

- Treatment of neutral-neutral scattering has not been adequately benchmarked, * Undetected problems may be preventing atom - molecule momentum transfer, * Is working correctly qualitatively,

- Set up test simulation with target fluxes enhanced by 10 ,

. $\Rightarrow P=12.5$ mTorr,

. Turn off neutral-neutral scattering: $P=6.3$,

- Turn off molecule-ion scattering: $P=5.4$. 


\section{Two Regimes of Operation}

(1) conductance limited leakage $\sim \mathrm{f}$, pressure $\sim$ const.

(2) flux limited

leakage $\sim$ const., pressure $\sim 1 / f$

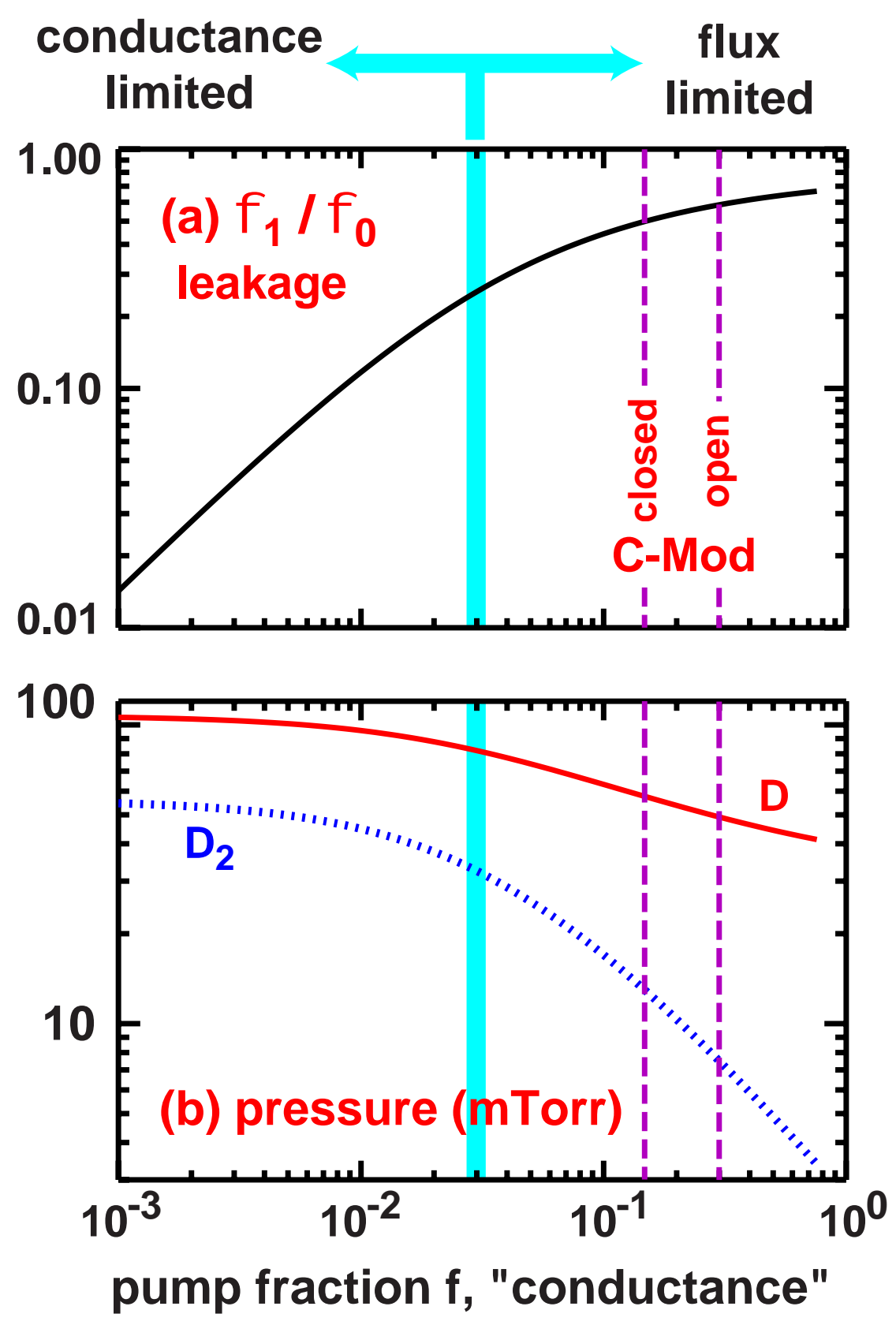

note: confirmed using DEGAS 2, see Stotler, P-2.59 
Total D Flux Vectors in $w=16$ mm Baseline Simulation

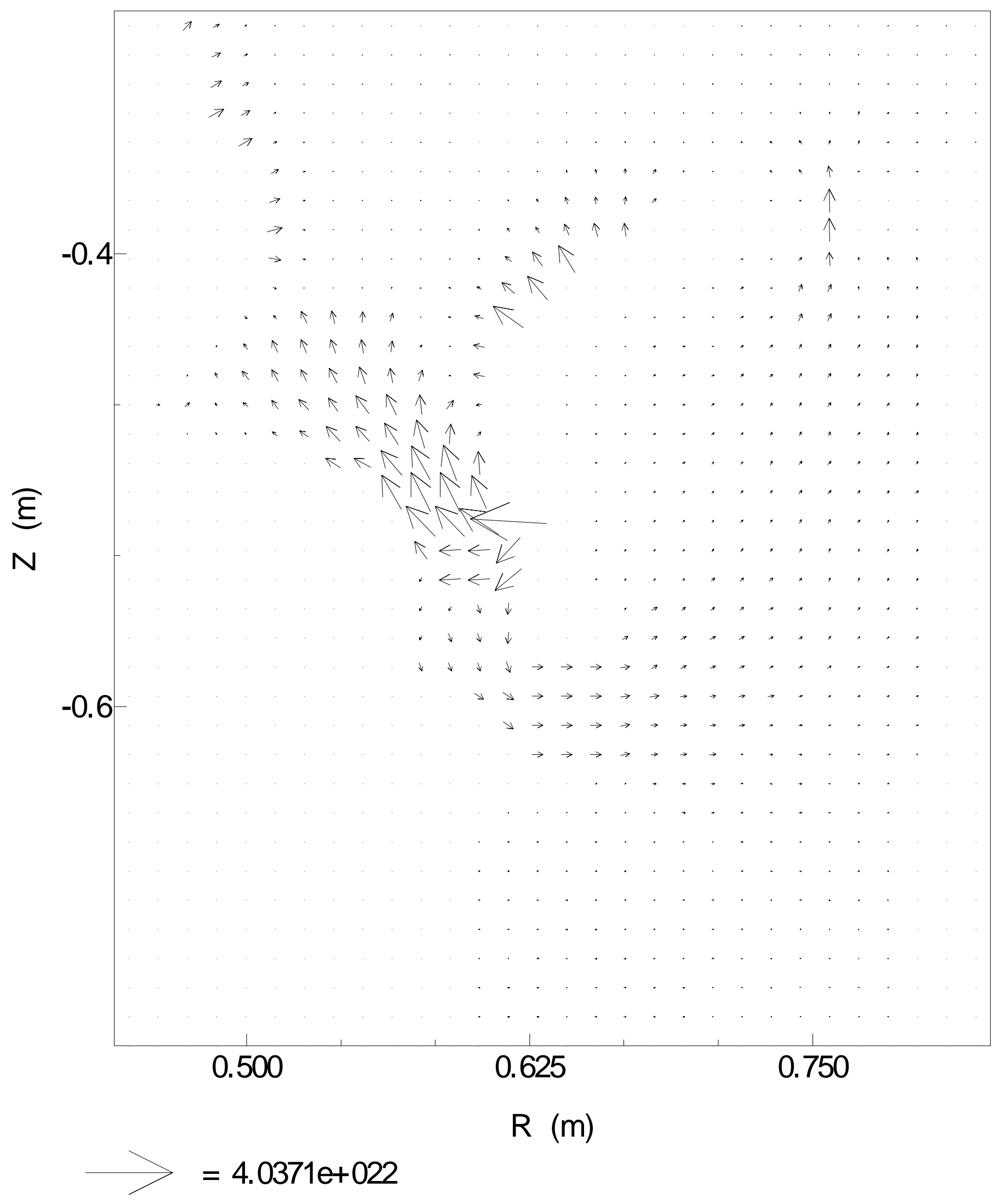



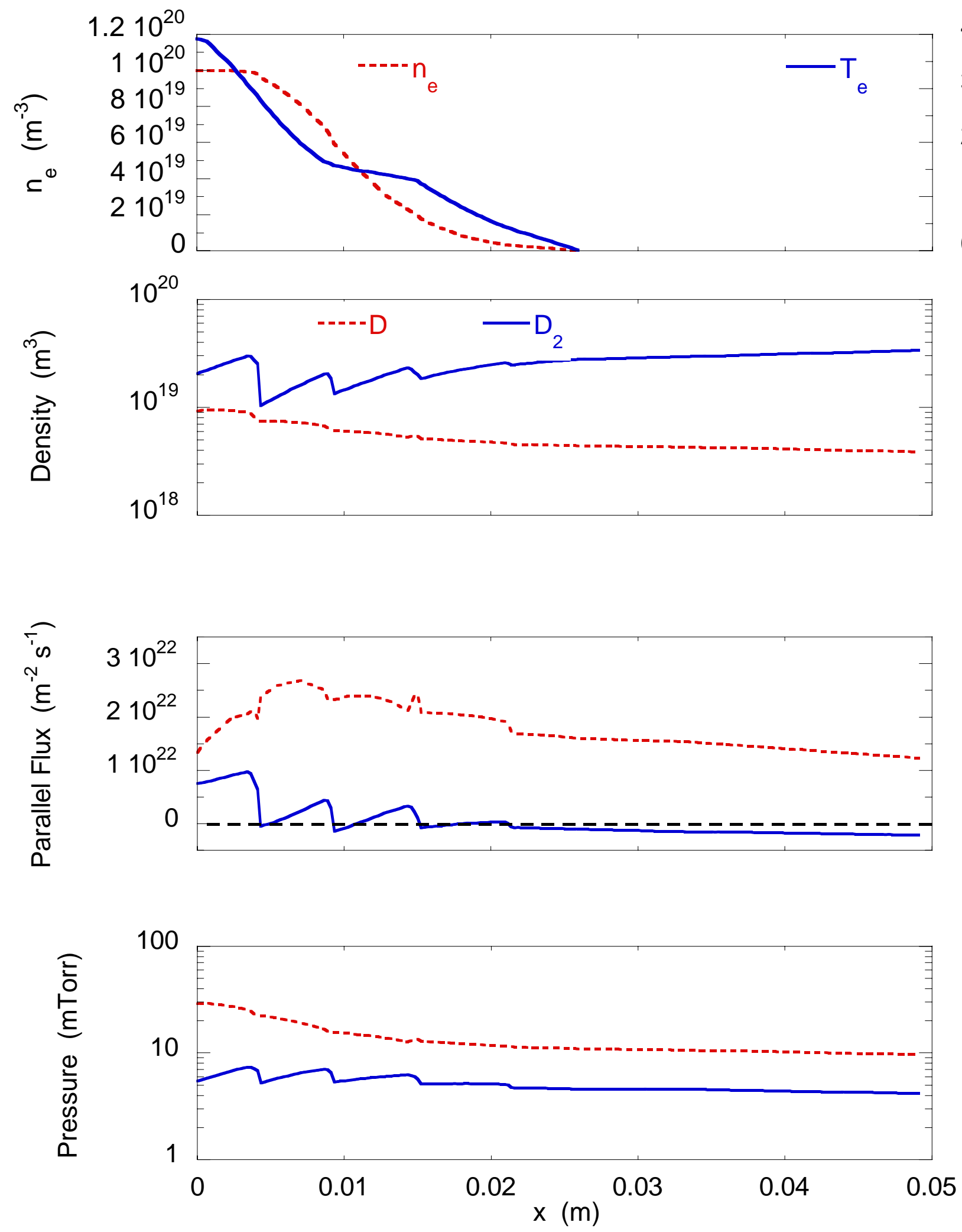


\section{Simple 1-D Model}
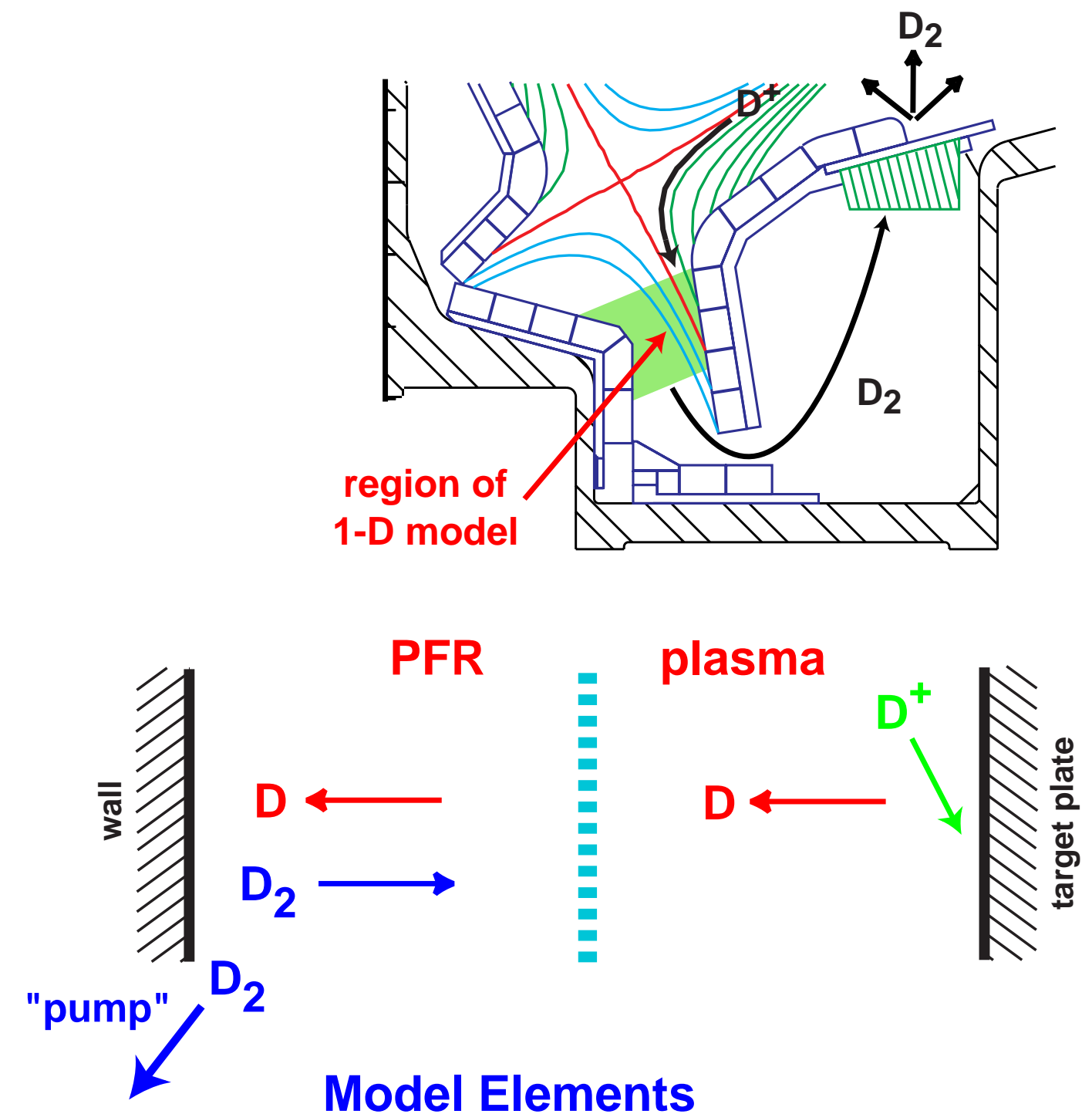

Model Elements

- atom, molecule: continuity, momentum

- plasma: ionization and CX

- PFR: momentum exchange between $D$ and $D_{2}$

Boundary Condition ("Pump Fraction" = f)

$$
f_{\text {pump }}=\frac{1}{4} n_{\text {mol }} \bar{c}_{\text {mol }} f
$$


Fig. 7

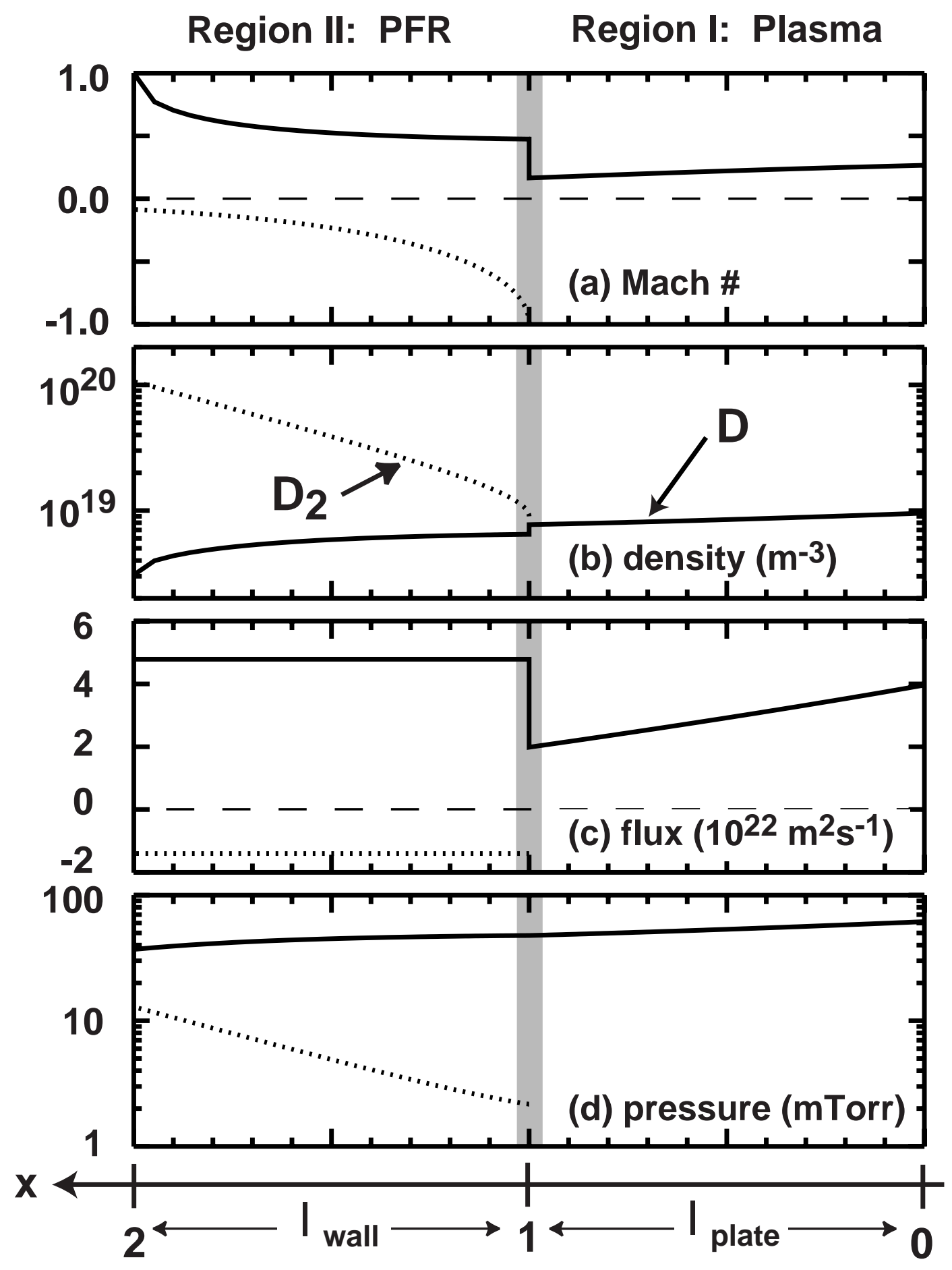




\section{CONCLUSIONS}

- Experimental results:

- Bypass strongly affects neutral pressure,

- But not bypass current,

- Plasma conditions and $\mathrm{D}_{\alpha}$ do not change.

- Principal result here:

- Reproduce same qualitative trends,

- Decreasing sensitivity of $\phi$ with $w$ $\Rightarrow$ some other process limiting flow,

- Conclude: divertor effectively open.

- Compare with 1-D model of (Pitcher 2000a):

- Replot data on log-log scale,

- Closely resembles Fig. 7 of (Pitcher 2000a),

- Quantitative differences arise,

- See also 1-D plots of DEGAS 2 data (bypass closed with capped $n, T$ ).

- "Flux limited" regime arises for $w \gtrsim 10 \mathrm{~mm}$,

- $\phi$ determined by competition between divertor ionization and escape through bypass,

- At large enough $w$ ionization \& CX limit $\phi$,

$-\Rightarrow$ open divertor.

- "Conductance limited" for $w<10 \mathrm{~mm}$,

- Linearly varying $\phi$ with $w$,

- Insensitive pressures. 


\section{BIBLIOGRAPHY}

Janev 1993 R. K. Janev and J. J. Smith, Atomic and Plasma-Material Interaction Data for Fusion (Supplement to the journal Nuclear Fusion) 4 (1993) 1.

Kanzleiter 1999 R. J. Kanzleiter, The Modeling and Impact of Neutral Elastic Collisions in LowTemperature High-Density Plasmas, Ph. D. Thesis, Rensselaer Polytechnic Institute (1999).

Krstic 1998 P. S. Krstic and D. R. Schultz, Atomic and Plasma-Material Data for Fusion 8 (1998) 1.

Niemczewski 1995 A. Niemczewski, Ph. D. thesis, MIT Plasma Fusion Center (1995).

Pitcher 1997 C. S. Pitcher and P. C. Stangeby, Plasma Phys. Controlled Fusion 39 (1997) 779.

Pitcher 2000 C. S. Pitcher et al., Phys. Plasmas 7 (2000) 1894.

Pitcher 20000a C. S. Pitcher et al., this conference.

Reiter 1997 D. Reiter et al., J. Nucl. Mater. 241-243 (1997) 342.

Shewchuk 1996 J. R. Shewchuk, in Applied Computational Geometry: Towards Geometric Engineering (M. C. Lin and D. Manocha, Eds.), Vol. 1148, Springer-Verlag, New York, 1996, p. 203.

Stotler 1992 D. P. Stotler and C. F. F. Karney, Contrib. Plasma Phys. 34 (1994) 392.

Stotler 1996 D. P. Stotler et al., Phys. Plasmas 3 (1996) 4084.

Stotler 2000 D. P. Stotler et al., Contrib. Plasma Phys. (2000).

Terry 1998 J. L. Terry et al., Phys. Plasmas 5 (1998) 1759.

Weisheit 1975 J. C. Weisheit, J. Phys. B: Atom. Molec. Phys. 8 (1975) 2556.

Umansky 1998 M. V. Umansky et al., Phys. Plasmas 5 (1998) 3373.

Note: This poster will be made available on the Web at:

http://w3 . pppl ·gov/degas2/

within two weeks of the end of the conference. 